\title{
Prevalence of Mental Disorders from Adolescence Through Early Adulthood in American Indian and First Nations Communities
}

\author{
Melissa Walls ${ }^{1}$ (D) Kelley J. Sittner ${ }^{2} \cdot$ Les B. Whitbeck $^{3} \cdot$ Kaley Herman $^{1}$ • \\ Miigis Gonzalez ${ }^{1}$ • Jessica H. L. Elm ${ }^{1}$ • Dane Hautala ${ }^{4} \cdot$ Melinda Dertinger $^{5}$ • \\ Dan R. Hoyt ${ }^{3}$
}

Published online: 8 June 2020

(C) Springer Science+Business Media, LLC, part of Springer Nature 2020

\begin{abstract}
Indigenous communities lack representation in psychiatric epidemiology despite disproportionate exposure to risk factors. We document the cumulative and 12-month prevalence of psychiatric disorders across the early life course among a sample of Indigenous young adults and compare prospective and retrospective reporting of lifetime mental disorders. This community-based participatory research includes data from 735 Indigenous people from 8 reservations/reserves. Personal interviews were conducted between 2002-2010 and 20172018 totaling 9 waves; diagnostic assessments of DSM-IV-TR alcohol abuse/dependence, marijuana use/dependence, other substance abuse/dependence, generalized anxiety disorder, major depressive disorder, dysthymic disorder, and attention deficit/hyperactivity disorder occurred at waves 1 (mean age $=11.1$ years), 4 (mean age $=14.3$ years), 6 (mean age $=$ 16.2 years), 8 (mean age $=18.3$ years), and 9 (mean age $=26.3$ years). Cumulative lifetime psychiatric disorders reached $77.3 \%$ and lifetime comorbidity $56.4 \%$ by wave 9 . Past-year prevalence and comorbidity at wave 9 were $28.7 \%$ and $6.7 \%$, respectively. Substance use disorders (SUDs) were most common with peak past-year prevalence observed when participants were on average 16.3 years old then declining thereafter. Trends in early life course psychiatric disorders in this study with Indigenous participants highlight cultural variations in psychiatric epidemiology including surprisingly low rates of internalizing
\end{abstract}

Melissa Walls

mwalls3@jhu.edu

1 Department of International Health; Center for American Indian Health, Johns Hopkins University, 1915 South Street, Duluth, MN 55812, USA

2 Department of Sociology, Oklahoma State University, Stillwater, OK, USA

3 Department of Sociology, University of Nebraska-Lincoln, Lincoln, NE, USA

4 Department of Psychiatry, University of Minnesota, Minneapolis, MN, USA

5 Department of Family Medicine \& Biobehavioral Health, University of Minnesota Medical School, Duluth Campus, Duluth, MN, USA 
disorders in the face of risk factors, disproportionately high rates of early-onset and lifetime SUD, and lower rates of past-year SUD in early adulthood compared with prior research.

Keywords American Indian · Native American · Psychiatric epidemiology · Mental disorder · Substance use disorder $\cdot$ Mental health $\cdot$ Substance use

American Indian (AI) and First Nations (i.e., Indigenous) communities withstand unequal burdens of historical and psychosocial stressors relative to non-Indigenous people (American Psychological Association 2016; Warne and Frizzell 2014). Included among these social determinants of health are ongoing discrimination and marginalization of Indigenous people, inequities in access to health care, and historical trauma, the accumulating, ongoing, and intergenerational transmission of psychological pain resulting from collective exposure to historically traumatic experiences (e.g., boarding schools, relocation programs) (American Psychological Association 2016; Warne and Frizzell 2014; King et al. 2009). These historically anchored and enduring factors result in substance misuse and mental health disparities for some Indigenous groups (King et al. 2009) that contribute heavily to heightened premature mortality rates (Shiels et al. 2017). Yet, we know little about psychiatric disorders among Indigenous people, particularly in the context of life course development. Without such data, targets for optimizing prevention and treatment are unclear, lessening the potential to improve health in Indigenous communities.

Among the data that do exist are findings from the Great Smoky Mountains Study (GSMS) where AI youth reported slightly lower prevalence of mental disorders compared with White youth in a shared geographic region (Costello et al. 1997). As they age through adolescence, however, Indigenous people appear to experience disproportionately high rates of substance use disorders (SUD) and related consequences (Costello et al. 1997; Whitbeck et al. 2014a). Importantly, rates of disorder vary tremendously across the exceptionally diverse tribal communities of North America (Beals et al. 2005; Koss et al. 2003), highlighting the value of within-culture studies.

Evidence from diverse populations suggests that early mental illness foretells later problems including adult psychiatric disorders (Federman et al. 1997; Harrington et al. 1990), but how this plays out in Indigenous communities is less clear. For instance, Indigenous youth may experience earlier onset SUD (Whitbeck et al. 2014a; Whitbeck et al. 2006a), and earlier intoxication has been linked to adolescent and early adult SUD in an urban AI sample (Henry et al. 2011); yet national data show high rates of AI alcohol abstinence in adulthood (Barnes et al. 2010; May 1996; Spicer et al. 2003). In addition, there appear to be relatively low levels of internalizing disorders like depression (Beals et al. 2005; Whitbeck et al. 2006b) among Indigenous adults despite high exposure to early life risks (Koss et al. 2003; Warne et al. 2017) and indicators of extreme psychological suffering (e.g., heightened youth suicide) (Indian Health Service 2015). These findings suggest unique patterns of disorder for Indigenous communities vis-à-vis non-Indigenous groups.

A more general limitation of the psychiatric epidemiological literature is a dearth of prospective studies of disorders in which individuals are re-assessed as they develop. Prospective studies are critical because retrospective approaches underestimate actual burden due to recall bias (Copeland et al. 2011; Takayanagi et al. 2014). For instance, lifetime disorder prevalence was doubled in prospective compared with that in retrospective data among 32year-olds (Moffitt et al. 2010) and two to twelve times greater in a sample that extending into older adulthood (Takayanagi et al. 2014). Prospective data spanning critical transitional 
periods of the life course (e.g., adolescence to adulthood) is especially sparse. The GSMS is a notable exception and included oversampling of Indigenous youth (Costello et al. 1996). While period (past-year) prevalence rates in the GSMS were relatively low at $13 \%$, the cumulative lifetime psychiatric disorder rate by age 21 was higher than $90 \%$ for the most frequently assessed study cohort (Copeland et al. 2011).

In sum, limited evidence hints at unique developmental experiences with mental disorders (i.e., lower internalizing disorders, earlier onset, and recovery from SUDs) for Indigenous people. There is little prospective data on psychiatric disorders generally and especially for Indigenous communities where burden of risk may be greatest and developmental rates of disorders are unknown. We contribute new information to these issues by documenting the cumulative and 12-month prevalence of select DSM-IV psychiatric disorders across the early life course among Indigenous young adults from a single cultural group. We also compare prospective and retrospective reporting of lifetime mental disorders.

\section{Methods}

Data are from Healing Pathways, a prospective, community-based participatory research (CBPR) project with four reservations in the Northern Midwest USA and four Canadian First Nations reserves, all representing a single Indigenous cultural group. We do not explicitly name the cultural background or sites involved in the study to honor community requests for anonymity. The study originated in the early 2000s with requests from tribal members to generate reliable data on the scope of mental health and substance use issues in their communities and to identify targets for substance use prevention and health promotion with resulting data. Community research councils (CRCs) are supported in each partnering site by project funding and are co-leaders on project design, implementation, and data interpretation activities; CRCs have also been active and creative in advising team efforts to translate scientific data into locally useful products. Outcomes of this process include tribally based presentations of research findings, creation of comprehensive technical reports shared with tribal leaders and service providers for local grant-writing and reporting needs, translation of manuscripts into infographics for community use, social media posts and newsletters to communicate study progress, and a YouTube channel to highlight major findings. In the most recent waves of data collection, we assessed participant views on the usefulness of the study and personal experiences associated with participation. Participants expressed overwhelming support for the study, project staff, and the potential impact of the work (e.g., "It has given me a sense of honor to help future generation of American Indians, because I hope to make positive improvement with others in my community and for my community," and "It reminds me of the importance to study cultures who are ignored by mainstream US culture. It reminds me how valuable and important my culture and ethnicity is to myself and future generations.").

The original study consisted of eight waves of data collected annually with a target adolescent and at least one primary caregiver beginning in 2002. Eligible adolescents were aged 10-12 years, enrolled tribal members, and living on or near (within 50 miles) the reservation/reserve. For more information on the study design and sampling procedure, see (Whitbeck et al. 2014b). Wave 9 data was collected from 2017 to 2018 with original target adolescents aged 24-27 years.

Diagnostic data were collected via computer-assisted personal interviews by trained tribal community interviewers. Participants received $\$ 40$ for waves $1-8$ and $\$ 50$ at wave 9 . Study 
procedures were collaboratively developed by community and university team members and approved by the university institutional review boards. All manuscripts are reviewed by reservation/reserve CRCs prior to submission for publication.

The sample consists of 735 adolescents who enrolled at baseline ${ }^{1}$ representing a response rate of $79.4 \%$. Diagnostic interviews were conducted at waves 1 (mean age $=11.1$ years), 4 (mean age $=14.3$ years), 6 (mean age $=16.2$ years), 8 (mean age $=18.3$ years), and 9 (mean age $=26.3$ years). Of the 735 baseline target adolescents, 659 were interviewed at wave 4 , 655 at wave 6,591 at wave 8, and 453 at wave 9; 27 adolescents passed away by wave 9, and 3 became ineligible due to disability or military service, and 3 did not complete a wave 9 diagnostic interview. After accounting for deceased and ineligible participants, retention rates were $90.5 \%, 90.1 \%, 81.9 \%$, and $64.3 \%$, respectively. Participants who remained in the study were no more likely to meet criteria for any of the mental disorders than those not interviewed at wave 9 , but those missed at wave 9 were more likely to be male.

The wave 9 sample was $42.3 \%$ male and $57.0 \%$ female $(0.7 \%$ reported non-binary gender identity). Two-thirds (67\%) reported living on a reservation/reserve. Approximately $28 \%$ did not finish high school, 39.5\% finished high school/earned a GED, 18.5\% had some college education, 9.1\% completed an associate's degree or trade school, and 3.8\% had a bachelor's degree. The mean annual personal income was $\$ 18,163$.

\section{Measures}

Psychiatric disorders were assessed with the Diagnostic Interview Schedule for Children-Revised (DISC-R) when the participants adolescents (waves 1-8) and the World Mental Health Survey Initiative Version of the World Health Organization Composite International Diagnostic Interview (CIDI) when participants were young adults (wave 9). Both instruments are fully structured and intended for use with lay interviewers (Kessler and Üstün 2004; Shaffer et al. 1993).

We assessed select DSM-IV-TR (American Psychiatric Association 2000) lifetime and past-year alcohol abuse and dependence, marijuana abuse and dependence, other drug abuse and dependence, generalized anxiety disorder (GAD), major depressive disorder (MDD), dysthymic disorder (DD), and attention deficit/hyperactivity disorder (ADHD). Adolescents and a caregiver were interviewed at waves 1 and 4, and the adolescent/young adult only thereafter. We present combined parent-adolescent reports for waves 1 and 4 based on reliability research on the DISC (Jensen et al. 1995) and common practice in epidemiological research (separate parent and adolescent reports have been presented elsewhere) (Whitbeck et al. 2008). We calculated cumulative lifetime rates for each disorder as the sum of the cases from the prior wave plus any new cases who met criteria in the current wave (Angold et al. 1996).

We included several other variables for use in this report, including two categories of disorder for each wave: (1) substance use disorder (alcohol abuse and dependence, marijuana abuse and dependence, other drug abuse and dependence) and (2) mood disorder (MDD and dysthymia). We also calculated the prevalence of participants who met the criteria for at least one disorder and for two or more disorders at each wave.

\footnotetext{
${ }^{1} N=11$ families enrolled at wave 1 of the study in two remote First Nations communities but were not reinterviewed at subsequent waves and thus are excluded from the current sampling frame (i.e., we did not attempt to contact them for inclusion in the longitudinal study).
} 


\section{Results}

\section{Cumulative Prevalence and Comorbidity}

By wave 9 (mean age $=26.3$ years), the cumulative lifetime prevalence of meeting diagnostic criteria for any mental disorder assessed in this study reached $77.3 \%$ and prevalence of any two or more disorders was $56.4 \%$ (Table 1). Lifetime disorder and comorbidity at wave 9 were higher among females than males; this difference was not statistically significant. Past-year prevalence of any disorder at wave 9 was $28.7 \%$ and past-year comorbidity $6.7 \%$. The highest observed estimates of past-year prevalence and comorbidity occurred at wave 4 when participants were on average 14.3 years of age.

\section{SUDs}

SUDs represented the highest prevalence of the observed cumulative lifetime disorders in this sample (68.2\%, wave 9). Peak rates of past-year SUDs (alcohol, marijuana) were seen at wave 6 (mean age $=16.2$ years). Nearly all of those meeting criteria for a SUD between waves 1-8 did so for alcohol or marijuana use disorders; rates of "other" SUDs hovered around 0-1\%. At wave 9, however, cumulative lifetime rates of other SUDs rose to $11.1 \%$ and $18.7 \%$ for abuse and dependence, respectively. Pastyear estimates of wave 9 "other" SUDs were $2.9 \%$ for abuse and $6 \%$ for dependence. The three most commonly endorsed other drugs of abuse and dependence were stimulants, opioids, and painkillers.

\section{Internalizing Disorders}

Lifetime estimates of GAD were significantly higher among females than males. Past-year GAD at wave 9 was $5.6 \%$ overall, representing the highest period estimate of GAD across longitudinal assessments. At wave 9, nearly one-quarter of the sample reported cumulative lifetime MDD and 7.3\% met past-year criteria. Cumulative lifetime DD was 5.6\%, and pastyear rates $1.1 \%$ at wave 9 .

\section{Attention Deficit/Hyperactivity Disorder}

Cumulative lifetime rates of ADHD at wave 9 were $18.4 \%$ with few cases meeting past-year criteria (2.9\%). Peak period estimates of ADHD were observed at wave $1(9.6 \%)$.

\section{Specific Comorbidity}

Past-year and lifetime wave 9 estimates of specific combinations of comorbid disorders are displayed in Table 2. Among the 94 participants meeting past-year criteria for SUDs (top half of table), $12.8 \%$ met criteria for GAD, and $11.7 \%$ met criteria for MDD. Two-thirds of participants met lifetime criteria for SUD (bottom half of the table). Of those, $27.7 \%$ met criteria for comorbid MDD, and $21.2 \%$ for ADHD. Rates of SUD with comorbid internalizing conditions were greater for females than males in both past-year and lifetime estimates. 


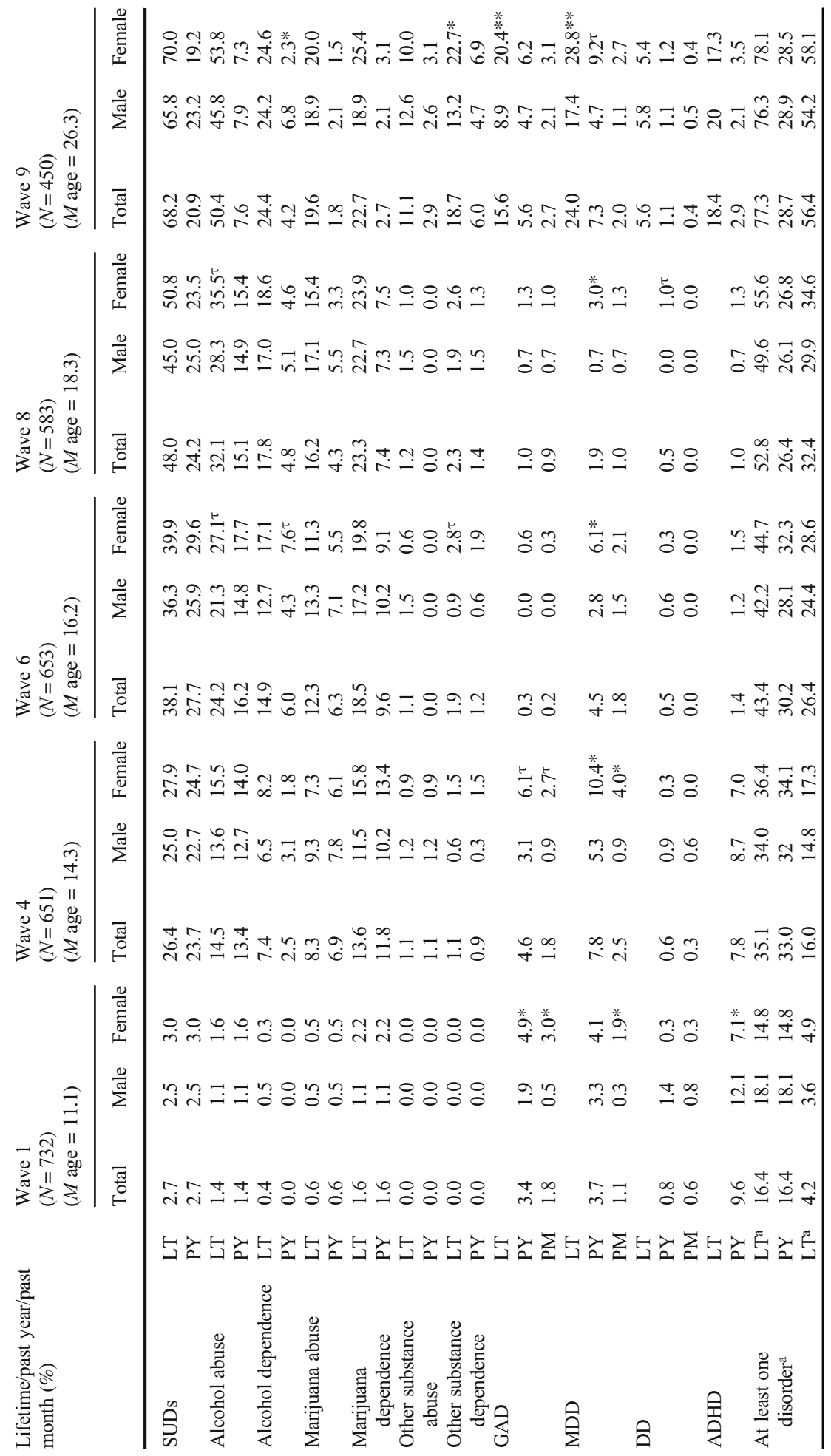




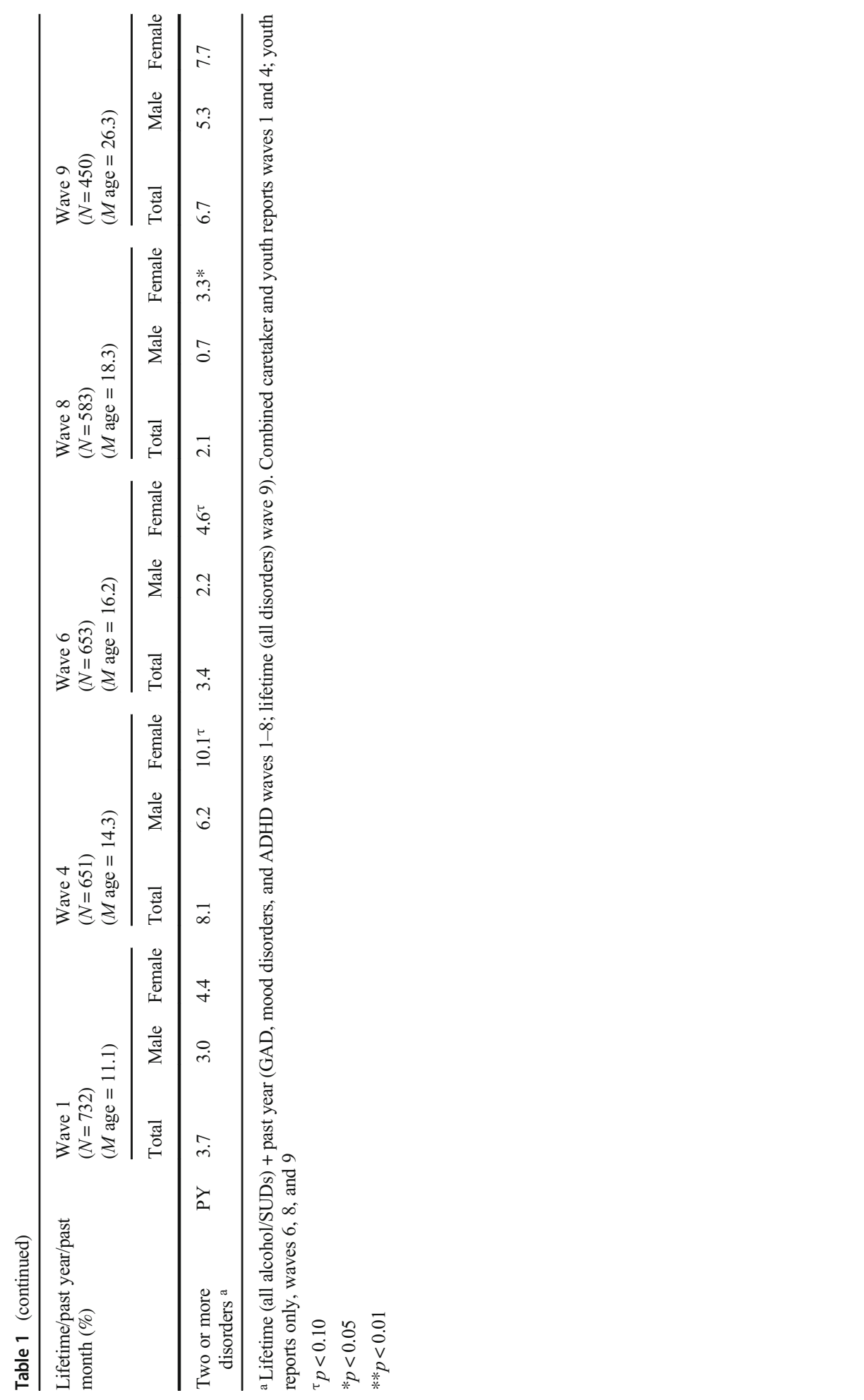


Table 2 Past-year and lifetime comorbidity for DSM-IV disorders, wave 9

\begin{tabular}{|c|c|c|c|c|c|c|}
\hline & \multicolumn{2}{|c|}{ Total $(n=450)$} & \multicolumn{2}{|c|}{ Males $(n=190)$} & \multicolumn{2}{|c|}{ Females $(n=260)$} \\
\hline & $n$ & $\%$ & $n$ & $\%$ & $n$ & $\%$ \\
\hline \multicolumn{7}{|c|}{ Past-year comorbidity } \\
\hline SUD & 94 & 20.9 & 44 & 23.2 & 50 & 19.2 \\
\hline With MDD & 11 & 11.7 & 1 & 2.3 & 10 & 20.0 \\
\hline With GAD & 12 & 12.8 & 5 & 11.4 & 7 & 14.0 \\
\hline With ADHD & 3 & 3.2 & 0 & 0.0 & 3 & 6.0 \\
\hline MDD & 33 & 7.3 & 9 & 4.7 & 24 & 9.2 \\
\hline With SUD & 11 & 33.3 & 1 & 11.1 & 10 & 41.7 \\
\hline With GAD & 12 & 36.4 & 4 & 44.4 & 8 & 33.3 \\
\hline With ADHD & 4 & 12.1 & 2 & 22.2 & 2 & 8.3 \\
\hline GAD & 25 & 5.3 & 9 & 4.7 & 16 & 6.2 \\
\hline With SUD & 12 & 48.0 & 5 & 55.6 & 7 & 43.8 \\
\hline With MDD & 12 & 48.0 & 4 & 44.4 & 8 & 50.0 \\
\hline With ADHD & 1 & 4.0 & 1 & 11.1 & 0 & 0.0 \\
\hline ADHD & 13 & 2.9 & 4 & 2.1 & 9 & 3.5 \\
\hline With SUD & 3 & 23.1 & 0 & 0.0 & 3 & 33.3 \\
\hline With MDD & 4 & 30.8 & 2 & 50.0 & 2 & 22.2 \\
\hline With GAD & 1 & 7.7 & 1 & 25.0 & 0 & 0.0 \\
\hline \multicolumn{7}{|c|}{ Lifetime comorbidity } \\
\hline SUD & 307 & 68.2 & 125 & 65.8 & 182 & 70.0 \\
\hline With MDD & 85 & 27.7 & 23 & 18.4 & 62 & 34.1 \\
\hline With GAD & 55 & 17.9 & 11 & 8.8 & 44 & 34.2 \\
\hline With ADHD & 65 & 21.2 & 25 & 20.0 & 40 & 22.0 \\
\hline MDD & 108 & 24.0 & 33 & 17.4 & 75 & 28.8 \\
\hline With SUD & 85 & 78.7 & 23 & 69.7 & 62 & 82.7 \\
\hline With GAD & 41 & 38.0 & 11 & 33.3 & 30 & 40.0 \\
\hline With ADHD & 31 & 28.7 & 9 & 27.3 & 22 & 29.3 \\
\hline GAD & 70 & 15.6 & 17 & 8.9 & 53 & 20.4 \\
\hline With SUD & 55 & 78.6 & 11 & 64.7 & 44 & 83.0 \\
\hline With MDD & 41 & 58.6 & 11 & 64.7 & 30 & 56.6 \\
\hline With ADHD & 24 & 34.3 & 7 & 41.2 & 17 & 32.1 \\
\hline ADHD & 83 & 18.4 & 38 & 20.0 & 45 & 17.3 \\
\hline With SUD & 65 & 78.3 & 25 & 65.8 & 40 & 88.9 \\
\hline With MDD & 31 & 37.3 & 9 & 23.7 & 22 & 48.9 \\
\hline With GAD & 24 & 28.9 & 7 & 18.4 & 17 & 37.8 \\
\hline
\end{tabular}

Although fewer participants met criteria for disorders other than SUD, large proportions of those who did met criteria for co-occurring disorders. The most frequent comorbid disorder among participants with past-year MDD $(n=33)$ was GAD (36.4\%) followed closely by SUD $(33.3 \%)$. MDD with GAD was more common for male compared with female participants, and MDD with SUD was higher for females than males. A sizeable proportion of the 108 participants who met lifetime criteria for MDD also had a lifetime history of SUD (78.7\%).

Comorbid SUD or MDD was present for roughly half of the 25 participants meeting criteria for past-year GAD. Among participants who met lifetime criteria for GAD $(n=70)$, over threequarters also had a lifetime history of SUD and 58.6\% MDD.

Of the thirteen participants meeting criteria for past-year ADHD, three (all females) also had past-year SUDs and four comorbid MDD. Among the 83 participants meeting lifetime ADHD criteria, $78.3 \%$ also reported lifetime SUDs. Lifetime ADHD combined with lifetime SUD among females represented the highest estimate of comorbidity observed in Table 2, reaching $88.9 \%$. 


\section{Retrospective and Cumulative Comparisons}

We compared cumulative rates of disorder to retrospective assessments at wave 9 and plotted these comparisons for SUDs (i.e., the disorders with lifetime reports at all waves of the study), any one disorder, and comorbidities in Fig. 1. Wave 9 retrospective assessment of any lifetime disorder underestimated prevalence by $13.5 \%$ compared with cumulative rates; comorbidity was underestimated by $22.8 \%$. Underestimation was most pronounced for marijuana dependence $(16.5 \%)$.

\section{Discussion}

This study provides new evidence of the cumulative, early life course burden of select psychiatric disorders and reinforces prior assertions that a majority of people will experience a mental disorder in their lifetime (Moffitt et al. 2010). Our prospective design is significant in that most existing community-based psychiatric studies rely on retrospective reports of lifetime experiences and yield severe underestimations of true disorder prevalence and uncertainty about mental health service needs. The sample includes Indigenous young adults, a group differentially exposed to historical and contemporary mental health risk factors but lacking representation in psychiatric epidemiological data. At an average of 26.3 years of age,
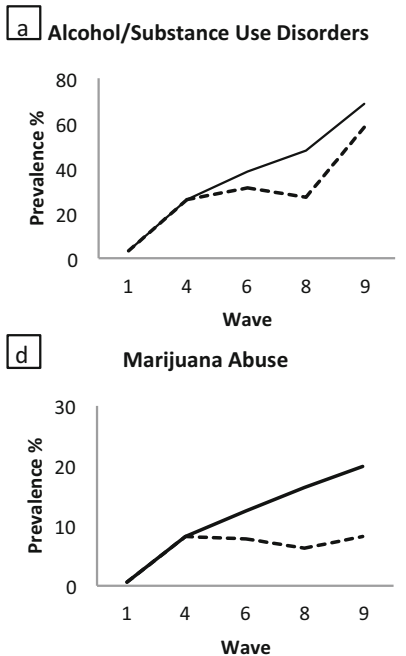

$\mathrm{g}$ Other Substance Dependence

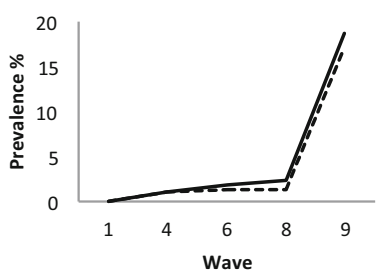

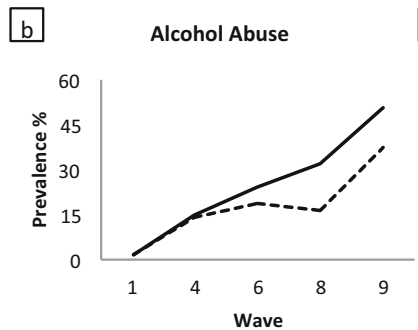

e

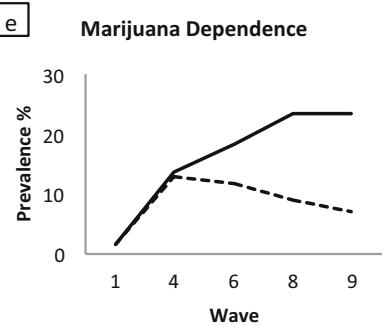

h

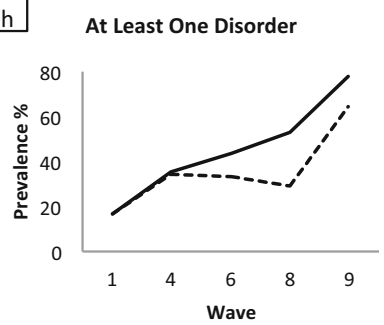

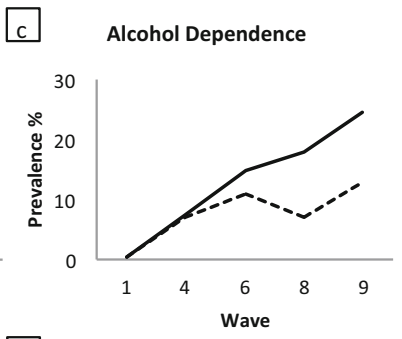

$f$ Other Substance Abuse

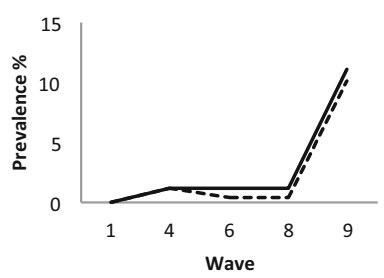

Two or More Disorders

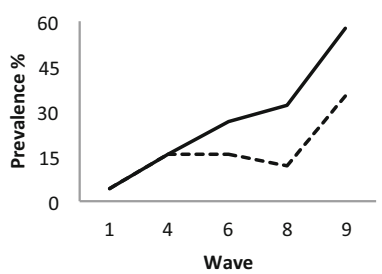

Fig. 1 Prevalence rates for cumulative and retrospective lifetime mental and substance use disorders 
cumulative lifetime rates of disorder in this study reached $77.3 \%$. Our estimate is lower than reports from a previous prospective design that included AIs where peak cumulative lifetime disorder was $90 \%$ (Copeland et al. 2011), though this discrepancy may be partially due to the fact that we assessed fewer disorders and accumulated fewer assessments than the comparison study. Cumulative comorbidity by wave 9 reached $56.4 \%$. Greater proportions of females compared with males who met criteria for past-year or lifetime GAD or MDD also met criteria for co-occurring SUD, although this trend was not consistently statistically significant. These gender differences were most pronounced at wave 9 and may signal the need for genderresponsive prevention and treatment initiatives for Indigenous youth and young adults.

Across all waves, SUDs were the most commonly occurring class of psychiatric disorders with cumulative wave 9 lifetime and past-year rates reaching $68.2 \%$ and $20.9 \%$, respectively. Lifetime alcohol use disorders (abuse $=50.4 \%$; dependence $=24.4 \%$ ) were comparatively higher than one cumulative estimate comparison (26\% alcohol use disorders) (Takayanagi et al. 2014), yet lower than the Dunedin longitudinal study (31.8\% alcohol dependence) (Moffitt et al. 2010). Marijuana abuse (19.6\%) and dependence (22.7\%) were similar in our sample to a cumulative estimate of $21 \%$ for cannabis use/dependence in the GSMS that included AIs (Copeland et al. 2017). Prior research suggests a median age of onset of 20 years for SUDs (Kessler et al. 2005). The GSMS sample of AI and White young adults demonstrated trajectories of cannabis use disorders across ethnicities that peaked in early adulthood (Copeland et al. 2017). It is therefore compelling that peak past-year rates for SUD in the current sample occurred at wave 6 when participants were an average of 16.2 years of age. The fact that a majority of lifetime cases of SUD can be accounted for prior to age 18 in these data corroborates previous reports of earlier onset and problematic substance use in some Indigenous communities (Roski et al. 1998; Stanley and Swaim 2015). Paradoxically, wave 9 (early adult) past-year SUD was lower than reported at adolescent assessments (waves 4, 6, 8) and not attributable to sample attrition (see Methods). Thus, research to identify factors relevant to recovery from SUD for Indigenous people across diverse tribal contexts is warranted.

A notable jump in "other" SUDs at wave 9 was largely accounted for by increases in abuse and dependence on painkillers, stimulants, and opioids. Early descriptions of the opioid crisis suggested the heaviest impacts among rural and White Americans (Dasgupta et al. 2018), and some national data demonstrate that AIs are less likely to die than Whites of opioid overdose (Scholl et al. 2019). Yet, AIs had a faster rate of increase in opioid-related deaths than did Whites between 1999 and 2015 (Joshi et al. 2018), and AIs in Minnesota (one state from which participants were drawn) were over 6 times more likely to die of opioid overdose than non-Natives between 2011 and 2015 (Scholl et al. 2019; Minnesota Department of Health 2018). These findings underscore the importance of local surveillance, cultural variations within ethnic groups, and attention to racial/ethnic disparities hidden by popular headlines. Existing evidence-based treatments for SUDs rely largely on evidence accumulated without Indigenous participants or perspectives, and tribal community members have expressed concerns about the cultural appropriateness of such interventions. The data presented here provide another compelling call for policymakers to take notice: Indian Health Service (IHS), tribal, urban, and AIAN research funding allocations to address AI needs should be increased and match community priorities for culturally grounded solutions.

Rates of cumulative internalizing disorders in this study sample were generally closer to those reported in prior longitudinal research. For instance, our cumulative estimations of lifetime MDD and GAD were $24 \%$ and $15.6 \%$ by age 26 . This compares to cumulative estimates for MDD of 13.1\% (Takayanagi et al. 2014) and 14.2\% for GAD (Moffitt et al. 
2010). A notable exception in this trend is a $41.4 \%$ estimated prevalence of MDD among 32year-olds in the Dunedin study (Moffitt et al. 2010). Our DSM-derived estimates of depression seem low given heightened exposure to risk factors and evidence that other indicators of psychological suffering like suicidality are disproportionately high in many Indigenous communities (Indian Health Service 2015). The validity of measures of internalizing symptoms across cultures has been previously critiqued (Nichter 2010), and our finding could be a signal that current operationalization of MDD misaligns with Indigenous expressions of distress (Kaufman et al. 2013; O’Nell 1996).

Given the lack of comparability between prospective and retrospective data, we further discuss our findings vis-à-vis existing epidemiological literature using retrospective reports and past-year prevalence. Wave 9 retrospective reports of lifetime disorder were $64.9 \%$. This finding is higher than results from the AI-SUPERPFP study including 15-54-year-old AI adults from two reservations (Southwest Tribe $=41.9 \%$; Northern Plains Tribe $=44.5 \%$ ) (Beals et al. 2005). Our estimate is on par with a national sample of AIANs (66.5\%) (Brave Heart et al. 2016), 10\% greater than reported for non-Hispanic Whites (57.5\%) (Brave Heart et al. 2016), and higher than both the widely cited prevalence rate of $46.4 \%$ for lifetime disorders from the National Comorbidity Survey sample overall, as well as the 18-29-year-old NCS cohort whose prevalence was estimated at 52.4\% (Kessler et al. 2005). The discrepancy in retrospective assessments of any lifetime disorder at wave 9 compared with calculated cumulative prevalence $(64.9 \%$ vs. $77.3 \%)$ is not nearly as large as the mismatch reported in previous literature ranging from two to twelve times higher (Takayanagi et al. 2014).

Nearly $30 \%$ of our sample met criteria for wave 9 past-year disorders compared with $21 \%$ (Southwest) and 24.3\% (Northern Plains) of AI-SUPERPFP participants (Beals et al. 2005), 43.4\% (women) and $41.5 \%$ (men; Axis 1 disorders only) of AIs nationally (Brave Heart et al. 2016), and $26.2 \%$ of the US general population (Kessler et al. 2005). Similar to lifetime reports, past-year SUDs were the most commonly reported class of disorders among current study participants.

In sum, the cumulative data reveal generally similar or, in some cases, lower rates of overall psychiatric disorder in this sample of Indigenous young adults relative to other prospective studies. Alternatively, comparisons of retrospectively reported lifetime disorder suggest greater burden of disorder in this sample than in other similar study designs with non-Indigenous people, underscoring the importance of longitudinal assessments to understand true prevalence and trajectories of mental health problems across communities. At wave 9, overall rates of past-year disorder were comparable with previous research. In total, overall prevalence of disorder appears similar in this sample to other epidemiological surveys; yet, a critical finding centers around the disproportionately high rates of lifetime SUD in this study relative to other reports. For example, wave 9 lifetime alcohol abuse reached an accumulated rate of $50.4 \%$ (37.3\% retrospective report) compared with $12.8 \%$ for $18-29$-year-olds in the National Epidemiologic Survey on Alcohol and Related Conditions (NESARC) (Hasin and Grant 2015). Past-year rates of alcohol abuse, however, were nearly identical between the young Indigenous adults in this study (7.6\%) and NESARC estimates (7.0\%), suggesting a paradoxically high rate of recovery despite early-onset (adolescent) SUD.

\section{Limitations and Future Directions}

We assessed select disorders due to resource limitations and efforts to reduce participant burden. Thus, overall cumulative prevalence of lifetime disorder experiences may be larger than estimated. 
Measurement validity is a concern in research outside of Euro-middle-class contexts, with special considerations for interpreting research with Indigenous communities (Walls et al. 2017). Diagnostic interview schedules like those used in this study rely on "rigid rules" and highly standardized question sets with the absence of professional clinical expertise in the diagnostic process (Wakefield and Schmitz 2009). Diagnostic dichotomies (yes/no) may overshadow nuances of distress more evident in continuous measures. Roughly $30 \%$ of the young adults in this study met past-year criteria for a wave 9 disorder, $10 \%$ less than previously reported by their own adult caregivers (Whitbeck et al. 2006b) and suggesting possible resilience across generations. Furthermore, 59.2\% of the young adults in this study are current parents/caregivers, and 59\% report desire to have/have more children. An important future investigation involves intergenerational stability and/or desistance of mental disorders for the current cohort of participants to identify those factors that create opportunities for breaking intergenerational cycles of mental health problems.

\section{Conclusion}

There are several unique findings in this study relative to prior reporting on psychiatric disorders over the life course. First, discrepancies between retrospective and prospective reports were not as large as observed in previous longitudinal designs. Whether these unique patterns are common to other Indigenous cultural groups remains an empirical question. Second, SUDs were the most commonly reported disorders in our sample, whereas internalizing disorders are the highest-ranking diagnostic category in many general population studies. Relatedly, and third, compared with non-Indigenous study samples, peak periods of SUD emerged earlier for these Indigenous participants. Fourth, declines in rates of SUD seem to occur for these Indigenous participants during periods of heightened risk identified in non-Indigenous samples (i.e., early adulthood) (Hasin and Grant 2015). These findings thus provide compelling evidence of cultural variation in psychiatric epidemiology, targets for early SUD prevention, and intimate important lessons that are to be learned from Indigenous communities regarding early life course recovery from psychiatric disorders, particularly SUDs.

Acknowledgments The Healing Pathways team includes David Bruyere, Laura Bruyere, Annabelle Jourdain, Priscilla Simard, Trisha Bruyere, Jake Becker, Laureen Bruyere, Frances Whitfield, GayeAnn Allen, Tina Handeland, Victoria Soulier, Bagwajikwe Madosh, Betty Jo Graveen, Clinton Isham, Carol Jenkins, Bill Butcher Jr., Delores Fairbanks, Devin Fineday, Bernadette Gotchie, Gloria Mellado, Christina Howard Marilyn Bowstring, Gary Charwood, Gina Stender, Roberta Roybal, Jim Bedeau, Kathy Dudley, Geraldine Brun, June Holstein, Frances Miller, Brenna Pemberton, Ed Strong, Barbara Thomas, Charity Prentice-Pemberton, FaLeisha Jourdain, Penny King, Valerie King, Linda Perkins, Christie Prentice, Gabe Henry, Howard Kabestra, Dallas Medicine, Glenn Cameron, Jackie Cameron, Gerilyn Fisher, Virginia Pateman, Irene Scott, Cindy McDougall, Whitney Accobee Celeste Cloud, Pat Moran, Stephanie Williams, Natalie Bergstrom, Bonnie Badboy, Elizabeth Kent, Sue Trnka, and Laurie Vilas.

Funding Information The research reported in this manuscript was supported by the National Institute on Drug Abuse of the National Institutes of Health under Award Number R01 DA039912 (M. Walls, PI). The content is solely the responsibility of the authors and does not necessarily represent the official views of the National Institutes of Health.

\section{Compliance with Ethical Standards}

Conflict of Interest The authors declare that they have no conflict of interest. 


\section{References}

American Psychiatric Association. (2000). Diagnostic and statistical manual of mental disorders: DSM-IV-TR. Washington, DC: American Psychiatric Association.

American Psychological Association (2016). Stress in America: the impact of discrimination. Stress in America Survey.

Angold, A., Erkanli, A., Costello, E. J., \& Rutter, M. (1996). Precision, reliability and accuracy in the dating of symptom onsets in child and adolescent psychopathology. Journal of Child Psychology and Psychiatry, 37, 657-664. https://doi.org/10.1111/j.1469-7610.1996.tb01457.x.

Barnes, P. M., Adams, P. F., \& Powell-Griner, E. (2010). Health Characteristics of the American Indian or Alaska Native Adult Population: United States, 2004-2008. Hyattsville: Centers for Disease Control, National Center for Health Statistics.

Beals, J., Manson, S. M., Whitesell, N. R., Spicer, P., Novins, D. K., \& Mitchell, C. M. (2005). Prevalence of DSM-IV disorders and attendant help-seeking in 2 American Indian reservation populations. Archives of General Psychiatry, 62, 99-108. https://doi.org/10.1001/archpsyc.62.1.99.

Brave Heart, M. Y. H., Lewis-Fernández, R., Beals, J., Hasin, D. S., Sugaya, L., Wang, S., Grant, B. F., \& Blanco, C. (2016). Psychiatric disorders and mental health treatment in American Indians and Alaska Natives: results of the National Epidemiologic Survey on Alcohol and Related Conditions. Social Psychiatry and Psychiatric Epidemiology, 51, 1033-1046. https://doi.org/10.1007/s00127-016-1225-4.

Copeland, W. E., Shanahan, L., Costello, E. J., \& Angold, A. (2011). Cumulative prevalence of psychiatric disorders by young adulthood: a prospective cohort analysis from the Great Smoky Mountains Study. Journal of the American Academy of Child and Adolescent Psychiatry, 50, 252-261. https://doi.org/10.1016 /j.jaac.2010.12.014.

Copeland, W. E., Hill, S., Costello, E. J., \& Shanahan, L. (2017). Cannabis use and disorder from childhood to adulthood in a longitudinal community sample with American Indians. Journal of the American Academy of Child and Adolescent Psychiatry, 56, 124-132.

Costello, E. J., Angold, A. A., Burns, B. J., et al. (1996). The Great Smoky Mountains Study of youth. Goals, design, methods, and the prevalence of DSM-III-R disorders. Archives of General Psychiatry, 53, 11291136.

Costello, E. J., Farmer, E. M. Z., Angold, A., et al. (1997). Psychiatric disorders among American Indian and white youth in Appalachia: the Great Smoky Mountains Study. American Journal of Public Health, 87, 827-832.

Dasgupta, N., Beletsky, L., \& Ciccarone, D. (2018). Opioid crisis: no easy fix to its social and economic determinants. American Journal of Public Health, 108, 182-186. https://doi.org/10.2105 /AJPH.2017.304187.

Federman, E. B., Costello, E. J., Angold, A., Farmer, E. M., \& Erkanli, A. (1997). Development of substance use and psychiatric comorbidity in an epidemiologic study of white and American Indian young adolescents the Great Smoky Mountains Study. Psychology of Addictive Behaviors, 44, 69-78.

Harrington, R., Fudge, H., Rutter, M., Pickles, A., \& Hill, J. (1990). Adult outcomes of childhood and adolescent depression: I. psychiatric status. Archives of General Psychiatry, 47, 465-473. https://doi.org/10.1001 /archpsyc.1990.01810170065010.

Hasin, D. S., \& Grant, B. F. (2015). The National Epidemiologic Survey on Alcohol and Related Conditions (NESARC) waves 1 and 2: review and summary of findings. Social Psychiatry and Psychiatric Epidemiology, 50, 1609-1640. https://doi.org/10.1007/s00127-015-1088-0.

Henry, K. L., McDonald, J. N., Oetting, E. R., et al. (2011). Age of onset of first alcohol intoxication and subsequent alcohol use among urban American Indian adolescents. Psychol Addict Behav J Soc Psychol Addict Behav, 25, 48-56. https://doi.org/10.1037/a0021710.

Indian Health Service. (2015). Trends in Indian health: 2014 Edition. Rockville: Indian Health Service.

Jensen, P., Roper, M., Fisher, P., Piacentini, J., Canino, G., Richters, J., Rubio-Stipec, M., Dulcan, M., Goodman, S., \& Davies, M. (1995). Test-retest reliability of the diagnostic interview schedule for children (DISC 2.1) parent, child, and combined algorithms. Archives of General Psychiatry, 52, 61-71.

Joshi, S., Weiser, T., \& Warren-Mears, V. (2018). Drug, opioid-involved, and heroin-involved overdose deaths among American Indians and Alaska Natives — Washington, 1999-2015. MMWR. Morbidity and Mortality Weekly Report, 67, 1384-1387. https://doi.org/10.15585/mmwr.mm6750a2.

Kaufman, C. E., Beals, J., Croy, C., Jiang, L., Novins, D. K., \& The AI-SUPERPFP Team. (2013). Multilevel context of depression in two American Indian tribes. Journal of Consulting and Clinical Psychology, 81, 1040-1051. https://doi.org/10.1037/a0034342.

Kessler, R. C., \& Üstün, T. B. (2004). The World Mental Health (WMH) survey initiative version of the World Health Organization (WHO) composite international diagnostic interview (CIDI). International Journal of Methods in Psychiatric Research, 13, 93-121. https://doi.org/10.1002/mpr.168. 
Kessler, R. C., Chiu, W. T., Demler, O., \& Walters, E. E. (2005). Prevalence, severity, and comorbidity of 12month DSM-IV disorders in the National Comorbidity Survey Replication. Archives of General Psychiatry, 62, 617-627. https://doi.org/10.1001/archpsyc.62.6.617.

King, M., Smith, A., \& Gracey, M. (2009). Indigenous health part 2: the underlying causes of the health gap. The Lancet, 374, 76-85. https://doi.org/10.1016/S0140-6736(09)60827-8.

Koss, M. P., Yuan, N. P., Dightman, D., Prince, R. J., Polacca, M., Sanderson, B., \& Goldman, D. (2003). Adverse childhood exposures and alcohol dependence among seven Native American tribes. American Journal of Preventive Medicine, 25, 238-244. https://doi.org/10.1016/S0749-3797(03)00195-8.

May, P. A. (1996). Overview of alcohol abuse epidemiology for American Indian populations. In G. D. Sandefur, R. R. Rindfuss, \& B. Cohen (Eds.), Changing numbers, changing needs: American Indian demography and public health (pp. 235-256). Washington, DC: National Academy Press.

Minnesota Department of Health. (2018). Race rate disparity in drug overdose deaths. St. Paul: Minnesota Department of Health.

Moffitt, T. E., Caspi, A., Taylor, A., Kokaua, J., Milne, B. J., Polanczyk, G., \& Poulton, R. (2010). How common are common mental disorders? Evidence that lifetime prevalence rates are doubled by prospective versus retrospective ascertainment. Psychological Medicine, 40, 899-909. https://doi.org/10.1017 /S0033291709991036.

Nichter, M. (2010). Idioms of distress revisited. Culture, Medicine and Psychiatry, 34, 401-416. https://doi. org/10.1007/s11013-010-9179-6.

O’Nell, T. D. (1996). Disciplined hearts: history, identity, and depression in an American Indian community. Berkeley: University of California Press.

Roski, J., Perry, C., McGovern, P., et al. (1998). Psychosocial factors associated with alcohol use among young adolescent American Indians and Whites. Journal of Child \& Adolescent Substance Abuse, 7, 1-18. https://doi.org/10.1300/J029v07n02_01.

Scholl, L., Seth, P., Kariisa, M., Wilson, N., \& Baldwin, G. (2019). Drug and opioid-involved overdose deaths - United States, 2013-2017. MMWR. Morbidity and Mortality Weekly Report, 67, 1419-1427. https://doi. org/10.15585/mmwr.mm6751521e1.

Shaffer, D., Schwab-Stone, M., Fisher, P., et al. (1993). The diagnostic interview schedule for children-revised version (DISC-R): I. preparation, field testing, interrater reliability, and acceptability. Journal of the American Academy of Child and Adolescent Psychiatry, 32, 643-650.

Shiels, M. S., Chernyavskiy, P., Anderson, W. F., Best, A. F., Haozous, E. A., Hartge, P., Rosenberg, P. S., Thomas, D., Freedman, N. D., \& de Gonzalez, A. B. (2017). Trends in premature mortality in the USA by sex, race, and ethnicity from 1999 to 2014: an analysis of death certificate data. The Lancet, 389, 1043-1054. https://doi.org/10.1016/S0140-6736(17)30187-3.

Spicer, P., Beals, J., Croy, C. D., Mitchell, C. M., Novins, D. K., Moore, L., \& Manson, S. M. (2003). The prevalence of DSM-III-R alcohol dependence in two American Indian populations. Alcoholism, Clinical and Experimental Research, 27, 1785-1797. https://doi.org/10.1097/01.ALC.0000095864.45755.53.

Stanley, L. R., \& Swaim, R. C. (2015). Initiation of alcohol, marijuana, and inhalant use by American-Indian and white youth living on or near reservations. Drug and Alcohol Dependence, 155, 90-96. https://doi. org/10.1016/j.drugalcdep.2015.08.009.

Takayanagi, Y., Spira, A. P., Roth, K. B., Gallo, J. J., Eaton, W. W., \& Mojtabai, R. (2014). Accuracy of reports of lifetime mental and physical disorders: results from the Baltimore Epidemiological Catchment Area Study. JAMA Psychiatry, 71, 273-280. https://doi.org/10.1001/jamapsychiatry.2013.3579.

Wakefield, J. C., \& Schmitz, M. F. (2009). The measurement of mental disorder. In T. L. Scheid \& T. N. Brown (Eds.), A handbook for the study of mental health: social contexts, theories, and systems (2nd ed., pp. 2045). Cambridge: Cambridge University Press.

Walls, M. L., Whitesell, N. R., Barlow, A., \& Sarche, M. (2017). Research with American Indian and Alaska Native populations: measurement matters. Journal of Ethnicity in Substance Abuse, 18, 1-21. https:/doi. org/10.1080/15332640.2017.1310640.

Warne, D., \& Frizzell, L. B. (2014). American Indian health policy: historical trends and contemporary issues. American Journal of Public Health, 104, S263-S267. https://doi.org/10.2105/AJPH.2013.301682.

Warne, D., Dulacki, K., Spurlock, M., Meath, T., Davis, M. M., Wright, B., \& McConnell, K. J. (2017). Adverse childhood experiences (ACE) among American Indians in South Dakota and associations with mental health conditions, alcohol use, and smoking. Journal of Health Care for the Poor and Underserved, 28, 15591577. https://doi.org/10.1353/hpu.2017.0133.

Whitbeck, L. B., Johnson, K. D., Hoyt, D. R., \& Walls, M. L. (2006a). Prevalence and comorbidity of mental disorders among American Indian children in the Northern Midwest. The Journal of Adolescent Health, 39, 427-434. https://doi.org/10.1016/j.jadohealth.2006.01.004. 
Whitbeck, L. B., Hoyt, D., Johnson, K., \& Chen, X. (2006b). Mental disorders among parents/caretakers of American Indian early adolescents in the Northern Midwest. Social Psychiatry and Psychiatric Epidemiology, 41, 632-640. https://doi.org/10.1007/s00127-006-0070-2.

Whitbeck, L. B., Yu, M., Johnson, K. D., Hoyt, D. R., \& Walls, M. L. (2008). Diagnostic prevalence rates from early to mid-adolescence among indigenous adolescents: first results from a longitudinal study. Journal of the American Academy of Child and Adolescent Psychiatry, 47, 890-900.

Whitbeck, L. B., Sittner Hartshorn, K. J., Crawford, D. M., Walls, M. L., Gentzler, K. C., \& Hoyt, D. R. (2014a). Mental and substance use disorders from early adolescence to young adulthood among indigenous young people: final diagnostic results from an 8-year panel study. Social Psychiatry and Psychiatric Epidemiology, 49, 961-973. https://doi.org/10.1007/s00127-014-0825-0.

Whitbeck LB, Sittner Hartshorn KJ, Walls ML (2014b). Indigenous adolescent development : psychological, Social and Historical Contexts. Routledge/Taylor \& Francis Group.

Publisher's Note Springer Nature remains neutral with regard to jurisdictional claims in published maps and institutional affiliations. 